\title{
Market Volatility Prediction Based on Long- and Short-Term Memory Retrieval Architectures
}

\author{
Jie Yuan** \\ Iowa State University \\ Ames, USA \\ jieyuan@iastate.edu
}

\author{
Zhu Zhang* \\ Iowa State University \\ Ames, USA \\ zhuzhang@iastate.edu
}

\begin{abstract}
Predicting market volatility is a critical issue in financial market research and practice. Moreover, in natural language processing, how to effectively leverage long- and short-term event sequences to predict market volatility is still a challenge. Especially, applying traditional recurrent neural networks (RNNs) on an extremely long event sequence is infeasible due to the high time complexity and the limited capability of the memory units in RNNs. In this paper, we propose a new deep neural network-based architecture named Long- and Short-term Memory Retrieval (LSMR) architecture to forecast short-term and mid-term volatility. LSMR architecture consists of three separate encoders, a query extractor, a long-term memory retriever, and a volatility predictor. The query extractor and the long-term memory retriever compose a long-term memory retrieval mechanism that enables the LSMR to handle the extremely long event sequences. Experiments on our novel news dataset demonstrate the superior performance of our proposed models in predicting highly volatile scenarios, compared to existing methods in the literature.
\end{abstract}

\section{CCS CONCEPTS}

- Applied computing $\rightarrow$ Economics; • Computing methodologies $\rightarrow$ Neural networks.

\section{KEYWORDS}

market volatility, long-term memory, short-term memory, retrieval, LSTM, RIMs

\section{ACM Reference Format:}

Jie Yuan and Zhu Zhang. 2020. Market Volatility Prediction Based on Longand Short-Term Memory Retrieval Architectures. In ACM International Conference on AI in Finance (ICAIF '20), October 15-16, 2020, New York, NY, USA. ACM, New York, NY, USA, 8 pages. https://doi.org/10.1145/3383455. 3422545

\section{INTRODUCTION}

In finance, volatility is the degree of variation of a trading price series over time [1]. Market volatility is generally considered as a vital metric to evaluate the level of risk, and thus it plays a critical

${ }^{*}$ Both authors contributed equally to this research.



This work is licensed under a Creative Commons Attribution International 4.0 License ICAIF '20, October 15-16, 2020, New York, NY, USA

(C) 2020 Copyright held by the owner/author(s).

ACM ISBN 978-1-4503-7584-9/20/10.

https://doi.org/10.1145/3383455.3422545 rule in assessing the stock market risk and the pricing of derivative securities [21]. However, one of the significant challenges for the volatility prediction is the high stochasticity of the market.

Throughout the past several decades, a large number of research studies formulate volatility prediction as time series forecasting using historical data [7, 11, 17]. However, in reality, one of the significant stochasticity sources of the market is unstructured textual data, such as public news, financial reports, and social media $[14,18,20]$.

With recent advances in natural language processing (NLP) and deep learning, it has become possible to leverage unstructured textual data to predict market volatility. A large number of current textual-based models only leverage short-term information (e.g., several days' news or tweets) to predict the stock movement [6, 20,22]; they ignore the long-term dependencies existing in textual data. For instance, a previous event that happened several months or years ago may have a latent relationship with today's stock. Several works leverage both short-term and long-term events to predict stock movements (classification task) $[5,6]$. However, they might lose part of the long-term temporal information by using convolutional neural networks to process sequential data.

Distinct from the previous works, we formulate market volatility prediction as a regression task and seek to build on recurrent neural networks (RNNs) to further explore the utility of long-term memory for short-term and mid-term volatility forecasting. Nevertheless, one issue is that the RNNs will lose long-term information if the time series is exceptionally long. For example, suppose there is 30 news in one day, and we want to leverage the previous 90 days' news to make a prediction. The length of time series will be 2700; it is far more computationally expensive and infeasible to apply RNN on such a long time series. To alleviate this issue and capture the long-term information efficiently meanwhile taking advantage of the short-term information, we propose a Long- and Short-term Memory Retrieval (LSMR) architecture in which a long-term memory retrieval mechanism compresses long-term memory into $K$ sub-sequences by retrieving from long-term memory and the $K$ sub-sequences not only consist of the essential long-term information but relatively short. Thus, it becomes feasible that RNNs can capture long-term information from extremely long memory by performing RNNs on the $K$ event sub-sequences.

Moreover, although RNNs, such as LSTM [12] and GRU [3], achieve great success on time series tasks, it is hard for one recurrent cell to capture the global dynamics (temporal patterns) and sequence information. Recently, [10] introduce a new neural recurrent model, called Recurrent Independent Mechanisms (RIMs), which performs better than LSTM [12] on multiple sequential tasks; 
the experiments show that RIMs can capture distinct temporal patterns in the data and improve generalization to settings where these patterns change. In this paper, to leverage and validate the power of RIMs, we apply RIMs and LSTM on volatility prediction tasks and compare the performances between RIMs and LSTM.

The contributions of this paper can be summarized as follows:

- We build a new news dataset from the Wall Street Journal (WSJ) and leverage it to predict the market volatility.

- We propose a long- and short-term memory retrieval (LSMR) architecture for the short-term and long-term volatility forecasting, and our LSMR-based models achieve superior performance in predicting highly volatile scenarios.

- To the best of our knowledge, it is the first time applying RIMs on the textual dataset for the volatility prediction, and indeed the experimental results show the potential capability of RIMs on processing the textual data.

\section{RELATED WORK}

One branch of research on forecasting market volatility is based on historical pricing data [8] [2] [15]. Due to the success of deep neural networks in other related domains, [17] propose a neural stochastic volatility model (NSVM) for volatility estimation and prediction. It is a stochastic recurrent neural network framework that can formulate the temporal dynamics of volatility over time. The NSVM only relies on the historical pricing data without incorporating additional sources of volatility information.

Another branch to predict market volatility is to take advantage of natural language resources, such as tweets, financial news, etc. [6] extract events from news text, model the influence of long, short term events by using conventional neural networks, and predict the stock price movements. [5] predict the stock price movement by adopting Open IE technology. [13] intimate the process of human processing chaotic online news and design a hybrid attention network to predict the stock trend. Most recently, [20] propose a model, called StockNet, to predict stock movement from tweets and historical prices by introducing a recurrent, continuous latent variable for better simulating stochastic. The above models are used to predict the stock price movements (classification task); in contrast, our goal is to alleviate a regression problem.

Recurrent neural networks, such as LSTM [12] and GRU [3], have been widely used in forecasting time series, however, a single recurrent cell cannot capture global sequential information. Recently, [10] propose Recurrent Independent Mechanisms (RIMs), which is a new recurrent architecture in which multiple RIMs can be active, interact, and share information. Each RIM has its independent dynamics operating by default, and occasionally to interact with other relevant RIMs and with selected elements of the encoded input. Unlike the gating mechanism applied to the inputs in the RNNs, RIMs control each module's input.

\section{BACKGROUND}

In this section, we introduce the architecture of LSTM and Recurrent Independent Mechanisms (RIMs).

\section{$3.1 \quad$ LSTM}

LSTM [12] is one of the prevalent recurrent neural models due to the long-term and short-term memory mechanism. It uses a cell state to maintain long-term memory, and the cell state $C$ at time step $t$ is updated in terms of an input gate $i_{t}$ and a forget gate $f_{t}$.

$$
\begin{gathered}
f_{t}=\sigma\left(W_{f} \cdot\left[h_{t-1}, x_{t}\right]+b_{f}\right) \\
i_{t}=\sigma\left(W_{i} \cdot\left[h_{t-1}, x_{t}\right]+b_{i}\right) \\
\hat{C}_{t}=\tanh \left(W_{C} \cdot\left[h_{t-1}, x_{t}\right]+b_{C}\right) \\
C_{t}=f_{t} \cdot C_{t-1}+i_{t} \cdot \hat{C}_{t}
\end{gathered}
$$

where $h_{t-1}$ is the hidden state at time step $t-1$, and $x_{t}$ is the input at time step $t . W_{f}, W_{i}, W_{c}, b_{f}, b_{i}$, and $b_{C}$ are model parameters. For the short-term memory, at time step $t$, an output gate $o_{t}$ controls the short-term memory.

$$
\begin{gathered}
o_{t}=\sigma\left(W_{o}\left[h_{t-1}, x_{t}\right]+b_{o}\right) \\
h_{t}=o_{t} \cdot \tanh \left(C_{t}\right)
\end{gathered}
$$

where $W_{o}$ and $b_{o}$ are model parameters.

However, if the input is hugely complex, one LSTM can not capture all the latent dynamics in the data. Therefore, [10] present that multiple independent recurrent units catch distinct patterns and communicate sparingly is a better mechanism to model dynamics.

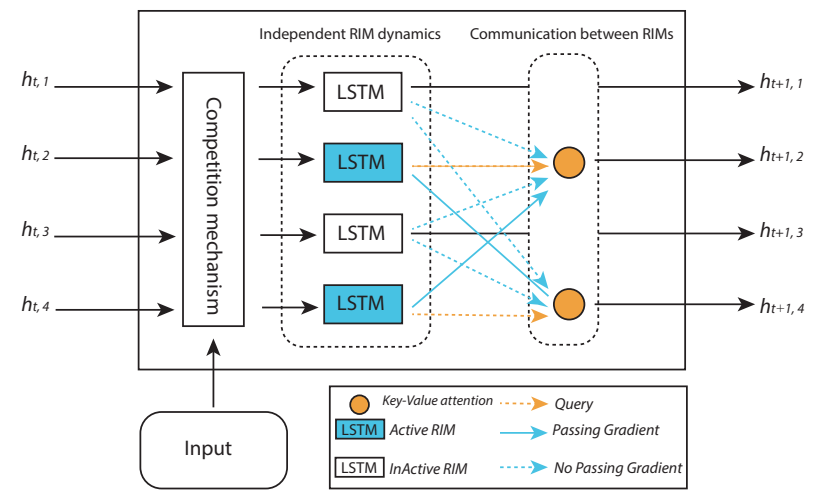

Figure 1: One step of Recurrent Independent Mechanisms (RIMS).

\subsection{Recurrent Independent Mechanisms (RIMs)}

In [10], a dynamic system can be composed of $K$ subsystems, which are denoted as Recurrent Independent Mechanisms (RIMs). RIMs operate with nearly independent transition dynamics, communicate only sparingly through the bottleneck of attention, and are only updated at time steps where they are most relevant. Each RIM, which can be implemented by using an LSTM or a GRU, is recurrent and has distinct functions that are learned automatically from data. For one single step of RIMs, it contains four stages: (1) Each RIM produces a query; (2) An attention-based competition mechanism is applied to select which RIMs to activate; (3) Activated RIMs follow their default transition dynamics while non-activated RIMs remain 
unchanged; (4) the RIMs sparsely communicate information with each other in terms of attention. A single step of RIMs is shown in Figure 1.

Produce a query (Stage 1): The state of RIM $k$ at time step $t$ can be denoted as $h_{t, k}$, where $t=1 \ldots T$, and then $Q_{k}=h_{t, k} W_{k}^{q}$ is the query of RIM $k$, where $W_{k}^{q}$ is a weight matrix which maps from the RIM's hidden state to its queries.

Competition mechanism (Stage 2): The goal of the competition mechanism is to dynamically select those RIMs for which the current input is relevant. The input at time $t$ is represented as $x_{t}$. Then, $x_{t}$ is concatenated with a row full of zeros $\varnothing$ to obtain $X$,

$$
X=x_{t} \oplus \varnothing
$$

Then, the attention score for RIM $k$ is defined as

$$
a_{k}^{i n}=\operatorname{softmax}\left(\frac{Q_{k} K^{T}}{\sqrt{d_{e}}}\right), k \in 1 \ldots K
$$

where $K=X W^{k}, W^{k}$ is a weight matrix; $d_{e}$ is the dimension of each key. Based on the softmax values, selecting top $k_{A}$ RIMs with the highest attention score (out of the total $K$ RIMs) to be activated and call them set $S_{t}$. Then, the new input for RIMs in $S_{t}$ is

$$
A_{k}^{i n}=a_{k}^{i n} X W^{v}
$$

where $W^{v}$ is a weight matrix; in practice, they use a multi-head attention technique.

Independent RIM dynamics (Stage 3): Stage 3 is to update the hidden states of individual RIMs. The hidden states of RIMs that the independent dynamics are applied are represented as $\widetilde{h}_{t, k}$. For the RIMs that are not activated, the hidden states remain unchanged,

$$
\widetilde{h}_{t, k}=h_{t, k}, \forall k \notin S_{t}
$$

The active RIMs are updated by

$$
\widetilde{h}_{t, k}=\operatorname{LSTM}\left(h_{t, k}, A_{k}^{i n} ; \theta_{k}^{D}\right), \forall k \in S_{t}
$$

where $\theta_{k}^{D}$ is the parameter of RIM $k, k \in S_{t}$. Each LSTM (RIM) has parameters $\theta_{k}$, which are shared across all time steps.

Communication between RIMs (Stage 4): The attention mechanism allows the sharing of information among the RIMs, specifically the activated RIMs, can be read from all other RIMs (activated or not).

$$
\begin{gathered}
Q_{t, k}=\widetilde{W}_{k}^{q} \widetilde{h}_{t, k}, \forall k \in S_{t} \\
K_{t, k}=\widetilde{W}_{k}^{e} \widetilde{h}_{t, k}, \forall k \\
V_{t, k}=\widetilde{W}_{k}^{v} \widetilde{h}_{t, k}, \forall k \\
h_{t+1, k}=\operatorname{softmax}\left(\frac{Q_{t, k}\left(K_{t,:}^{T}\right)}{\sqrt{d_{e}}} V_{t,:}\right)+\widetilde{h}_{t, k}, \forall k \in S_{t}
\end{gathered}
$$

where $\widetilde{W}_{k}^{q}, \widetilde{W}_{k}^{e}$, and $\widetilde{W}_{k}^{v}$ are weight matrices.

\section{MODEL FOR VOLATILITY PREDICTION}

In this section, we first formulate the problem of volatility prediction. Then, we introduce the long- and short-term memory retrieval (LSMR) architecture, which is composed of five components: Sentence encoder, Short-term event sequence encoder, Query extractor, Long-term memory retriever, Long-term event sequence encoder, and volatility predictor. The overall architecture is shown in Fig 2.

\subsection{Problem Formulation}

We aim at predicting the volatility of the S\&P 500 (SP) index, SPVt1, and $S P V t 22$, at stock market close time $t$ on a target trading day $d$ based on news titles in the Wall Street Journal. The volatility $(V t)$ is defined as the difference between anchor day's high and low price divide the average of anchor day's open and close prices.

The SPVt1 and SPVt22 are defined as follow:

$$
\begin{aligned}
& S P V t 1=\frac{\operatorname{Max}\left(\text { PriceHigh }_{t: t+1}\right)-\text { Min }_{\left(\text {PriceLow }_{t: t+1}\right)}}{\text { Avg } \left._{\text {PriceClose }} t-1, \text { PriceClose }_{t+1}\right)} \\
& \text { SPVt22 }=\frac{\operatorname{Max}\left(\text { PriceHigh }_{t: t+22}\right)-\operatorname{Min}\left(\text { PriceLow }_{t: t+22}\right)}{\text { Avg } \left._{\text {PriceClose }} t-1, \text { PriceClose }_{t+22}\right)}
\end{aligned}
$$

Formally, the problem of volatility prediction can be formalized as follows: given a target trading day $d$, a stock market close time $t_{d}$ on $d$ and a time window $W_{d}$, where $W_{d}=\left[t_{d}-\Delta h, t_{d}-1_{\text {hour }}\right]$ and $\Delta h$ is a fixed lag size. Then, a short-term memory can be denoted as $M_{\text {short }}=W_{d}$, and a long-term memory can be represented as $M_{\text {long }}=\left[W_{d-1 \text { day }}, W_{d-2 \text { days }}, \ldots, W_{d-n \text { days }}\right]$. In each time window $W_{d}$, there are $N$ news titles in $W_{d}$, and all titles in $W_{d}=$ $\left[N T_{1}, N T_{2}, \ldots, N T_{N}\right]$, and all titles in $W_{d}$ are sorted in accordance with Unix time. For each news title $N T_{n}, n \in[1, N]$, it contains $L$ tokens $T K, N T_{n}=\left[T K_{1}, T K_{2}, \ldots, T K_{L}\right]$. Our goal is to use the long-term and the short term memory to predict $S P V t_{1}$ and $S P V t_{22}$.

\subsection{Model Components}

Sentence encoder: We take advantage of pre-trained BERT ${ }^{1}$ [4] [19] to tokenize and encode news title $N T_{n}$ to a sequence of token embedding $N T_{n}^{E}=\left[T K_{1}^{E}, T K_{2}^{E}, \ldots, T K_{L}^{E}\right]$, where $T K_{l}^{E} \in \mathbb{R}^{1 \times d_{m}}, l \in$ $[1, L]$. We feed news title into pre-trained BERT and extract the last second hidden layer and then average the extracted layer to construct a news title vector $N T_{n}^{B E R T} \in \mathbb{R}^{1 \times d_{m}}$. Then, the shortterm memory can be denoted as $M_{\text {short }}^{E}=W_{d}^{E}$, where

$$
W_{d}^{E}=\left[N T_{1}^{B E R T}, N T_{2}^{B E R T}, \ldots, N T_{N}^{B E R T}\right]
$$

The long-term memory can be represented as

$$
M_{\text {long }}^{E}=\left[W_{d-1 \text { day }}^{E}, W_{d-2 \text { days }}^{E}, \ldots, W_{d-n \text { days }}^{E}\right]
$$

Short-term event sequence encoder: To capture the shortterm information; the $M_{\text {short }}^{E}$ is encoded by using recurrent neural network RNN (LSTM or RIMs):

$$
h_{\text {short }}=R N N\left(M_{\text {short }}^{E}\right)
$$

Since traditional RNNs can not deal with extremely long timeseries efficiently, we alleviate this issue by proposing a long-term memory retrieval mechanism. First, we leverage query extractor to extract the $k$ significant events (news titles) in terms of attention

${ }^{1}$ https://s3.amazonaws.com/models.huggingface.co/bert/bert-base-uncased-
pytorch_model.bin 


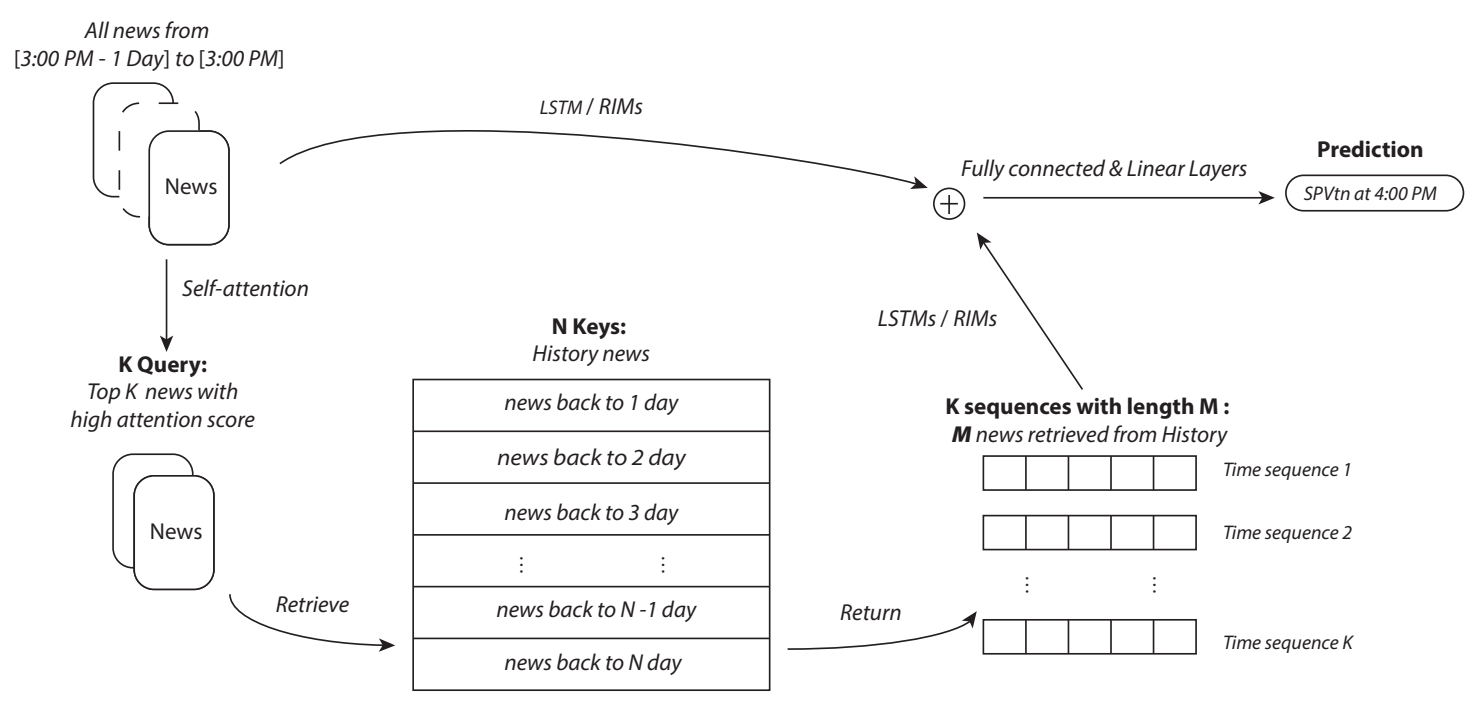

Figure 2: The Long- and Short-term Memory Retrieval (LSMR) architecture.

scores. Then, we perform long-term memory retriever on longterm memory and return $K$ event (sentences) sub-sequences with length $M$, which compresses long-term information into $K$ relative short event sequences. By leveraging the long-term memory retrieval mechanism, RNNs can be applied to $K$ event sub-sequences to efficiently capture long-term information.

Query extractor: Not all the news in $W_{d}$ has a high relationship with volatility, thus we aim to find the $K$ most significant event in $W_{d}$ by using a self-attention mechanism. We extract $K$ titles as queries from $W_{d}$, which have high self-attention scores:

$$
\begin{gathered}
\text { raw_score }=\tanh \left(\boldsymbol{W}_{2} \tanh \left(\boldsymbol{W}_{\mathbf{1}}\left(M_{\text {short }}^{E}\right)^{T}\right)+\boldsymbol{b}_{\mathbf{1}}\right)+\boldsymbol{b}_{2} \\
\text { att_score }=\operatorname{softmax}(\text { raw_score })
\end{gathered}
$$

where $\boldsymbol{W}_{\mathbf{1}}, \boldsymbol{W}_{2}, \boldsymbol{b}_{\mathbf{1}}$ and $\boldsymbol{b}_{2}$ are model parameters. $M_{\text {short }}^{E} \in \mathbb{R}^{N \times d_{m}}$ represents embeded short-term memory. raw_score, att_score $\epsilon$ $\mathbb{R}^{1 \times N}$.

$$
K-\text { queries }=\left\{N T_{i}^{\text {Embed }} \mid i \in \text { topK_index }(\text { att_score })\right\}
$$

where topK_index(att_score) returns the indices of top $\mathrm{K} N T_{i}^{E m b e d}$ which have high attention scores.

Long-term memory retriever: For each query out of $K$ queries, we use the query to retrieve a time sequences of titles from longterm memory. The intuition is that each query represents a significant event in $W_{d}$, we can find a similar event that happened in history, and there is an underlying pattern that is highly related to the current market. We leverage bi-linear matrix to mine the underlying long-term pattern, and the long-term memory retriever is defined as:

$$
\begin{aligned}
\text { scores }_{k}= & N T_{k}^{\text {Embed }} W_{3}\left(M_{\text {Long }}^{E}\right)^{T}, N T_{k}^{\text {Embed }} \in K-\text { queries } \\
& \text { seq_att_scores }_{k}=\operatorname{softmax}\left(\text { scores }_{k}\right), k \in K
\end{aligned}
$$

where $N T_{k}^{E m b e d}$ is $k_{t h}$ query in K-queries, $\boldsymbol{W}_{3} \in \mathbb{R}^{d_{m} \times d_{m}}$ is a bilinear matrix. Then, for each query $N T_{k}^{\text {Embed }} \in K$-queries, we can extract a time sequence with length $M$ from $M_{\text {Long }}$.

$$
\text { sequences }_{k}=\left\{N T_{i}^{\text {Embed }} \mid i \in \text { topK_index temp }(\text { seq_att_scores })\right\}
$$

where topK_index temp returns a sentence (news title) sequence in temporal order; sequences $k_{k} \in \mathbb{R}^{M \times d_{m}}$.

Long-term event sequence encoder: The long-term retriever returns $K$ event sequences; then, we perform RNN (LSTM or RIMs) on $K$ event sequences:

$$
h_{k}=R N N\left(\text { sequences }_{k}\right), k \in[1, \ldots, K]
$$

Volatility predictor: We leverage the concatenation of the $K$ encoded long-term event sequences $\left[h_{1} ; h_{2} ; \ldots ; h_{K}\right]$ and the encoded short-term event sequence $h_{\text {short }}$ to predict the volatility:

$$
Y=\boldsymbol{W}_{5}\left(\boldsymbol{W}_{4}\left[h_{1} ; h_{2} ; \ldots ; h_{K} ; h_{\text {short }}\right]+\boldsymbol{b}_{4}\right)+\boldsymbol{b}_{5}
$$

Where $\boldsymbol{W}_{\mathbf{4}}, \boldsymbol{W}_{\mathbf{5}}, \boldsymbol{b}_{\mathbf{4}}$ and $\boldsymbol{b}_{5}$ are model parameters, $Y$ is the volatility to predict, $\left[h_{1} ; h_{2} ; \ldots ; h_{K} ; h_{\text {short }}\right]$ is the concatenation of $\mathrm{K}$ hidden layers and $h_{\text {short }}$.

Finally, by using different recurrent unit (LSTM or RIMs), we obtain two models: Long- and Short-term Memory Retrieval LSTM (LSMR-LSTM) and Long- and Short-term Memory Retrieval RIMs (LSMR-RIMs).

\section{EXPERIMENTS}

In this section, we introduce our dataset for our application and evaluation metrics for assessing the performance of different models. Several baselines for volatility prediction are also presented.

\subsection{Data Collection}

In our experiments, we create a novel dataset from the Wall Street News. We use the volatility of S\&P 500 (SP) index from 01/02/2001 to $03 / 13 / 2018$ as our prediction target. We split them temporally, and 2,898 days from $01 / 02 / 2001$ to $07 / 10 / 2012$ are for training, 723 
days from $07 / 11 / 2012$ to $05 / 29 / 2015$ are for validation, and 702 days from $06 / 01 / 2015$ to $03 / 13 / 2018$ are for the test. Our task is to use the news to predict market volatility, and Wall Street Journal (WSJ) is a U.S. finance-focused newspaper, which has millions of news concerning the business. So, we crawl all the news in WSJ archive ${ }^{2}$ from 2001 to 2018 and finally obtain 699,290 news.

We observe that not all the news published in the WSJ is highly related to business. There is much noise in our crawled news dataset, and we also observe that all the news contains a article_type tag indicted which type of the news belongs to. We pre-process news dataset by computing the word cosine similarity between article_type with four types, Business, Economy, Stock and Market separately. article_type and four types are converted to word embedding pre-trained by Glove ${ }^{3}$. Then for each news, comparing the sum of four similarity scores with a threshold (the threshold is set to be 2.0), if the sum of similarity scores is less than the threshold, the news will be removed from the dataset. After preprocessing, 606,523 news is removed, and our final dataset has 92,767 news.

\subsection{Training setup}

In our experiments, we predefined the time lag $\Delta h=25$, the size of long-term memory $n=30$ or 90 , the number of news titles $N=30$ and the title length $L=20$. For the LSTM-based models, the dimension of the hidden layer of LSTM is 200, and we use two layers in LSTM. We use Xavier uniform initializer [9] to initialize parameters in LSTM. For RIMs-based models, the number of RIMs $K=4$, and the number of activated RIMs $K_{A}=2$. The dimension of the hidden state of individual RIM is 64. For the LSMR architecture, the number of queries $K=3$ in the query extractor component; the sequence length $M=20$ in long-term memory retriever. We train all the models with an Adam optimizer [16] with an initializing learning rate of $10^{-4}$, and the dropout rate is set to 0.2 . The batch size is 32 , the dimension of token embedding $d_{m}=768$, and the dimension of a fully connected layer is 200 . All the models run five times, and the hyper-parameters are tweaked on the validation dataset.

\subsection{Evaluation Metrics for Volatility Prediction}

Firstly, to evaluate the performance of our models and baselines on volatility prediction, we use MAPE, MAE, MSE (Errors) as evaluation metrics (lower errors are better).

Except that, in the financial field, investors care more about spikes (high volatility), so we classify target variables into two classes and convert the target variable $Y \geq \mu+\sigma$ to positive class and $Y<\mu+\sigma$ to negative class, where $\mu$ is the mean of the target variable, and $\sigma$ is the standard error of the target variable. Then, taking advantage of Precision, Recall, and F1-score (higher values are better) as metrics to evaluate the models on predicting spikes.

It should be noticed that the goals of the two sets of metrics are different. Errors are used to measure how well models fit the target variables, while Precision, Recall, and F1-value (P/R/F) are employed to evaluate how successful the models on predicting spikes. High $\mathrm{P} / \mathrm{R} / \mathrm{F}$ means more spikes can be predicted, meanwhile

\footnotetext{
$\overline{{ }^{2} \text { https://www.wsj.com/news/archive/ }}$

${ }^{3}$ http://nlp.stanford.edu/data/glove.42B.300d.zip
}

it will results in high Errors as well. In this paper, we pay more attention to the spikes prediction $(\mathrm{P} / \mathrm{R} / \mathrm{F})$.

The definition of MAPE, MAE, MSE, Precision, Recall, and F1value are as follows:

$$
\begin{gathered}
\operatorname{MAPE}(y, \hat{y})=\frac{100 \%}{N} \sum_{i=1}^{N}\left|\frac{y_{i}-\hat{y}_{i}}{y_{i}}\right| \\
\operatorname{MAE}(y, \hat{y})=\frac{1}{N} \sum_{i=1}^{N}\left|y_{i}-\hat{y}_{i}\right| \\
\operatorname{MSE}(y, \hat{y})=\frac{1}{N} \sum_{i=1}^{N}\left(y_{i}-\hat{y}_{i}\right)^{2}
\end{gathered}
$$

where $y_{i}$ is the actual value, $\hat{y}_{i}$ is the forecast value. $N$ is the number of samples.

$$
\text { Precision }=\frac{T P}{T P+F P}
$$

where TP denotes True Positive. If $Y \geq \mu+\sigma$ and $\hat{Y} \geq \mu+\sigma$, data point will be classified to TP. FP denotes False Positive, if $Y<\mu+\sigma$ and $\hat{Y} \geq \mu+\sigma$, data point will be classified to FP.

$$
\text { Recall }=\frac{T P}{T P+F N}
$$

where FN denotes False Negative. If $Y \geq \mu+\sigma$ and $\hat{Y}<\mu+\sigma$, the data point will be classified to FN.

$$
F 1-\text { value }=2 \cdot \frac{\text { Precision } \cdot \text { Recall }}{\text { Precision }+ \text { Recall }}
$$

F1-value is calculated based on Precision and Recall.

\subsection{Proposed Models and Baselines}

To make a detailed analysis of the utility of long-term memory and short-term memory, we construct two short-term based models and two long-term based models.

For short-term based models, we preprocess the input by splitting $W_{d}$ into 24 segments, and the time range of one segment is one hour. In each segment, the number of news titles is five, and the length of titles (the number of tokens) is 20 .

- Nested Attention Short-term Memory LSTM (NASM-LSTM): we introduce a nested attention mechanism to firstly aggregate the tokens weighted by assigned word-level attention values, then compute the weighted sum of sentences in the individual segment by using sentence-level attention values. After that, we obtain a temporal sequence with length 24 and apply LSTM on it. Finally, the last hidden layer of LSTM is feed into a fully connected layer and a linear layer to make predictions.

- Nested Attention Short-term Memory RIMs (NASM-RIMs): The LSTM in NASM-LSTM is replaced with RIMs.

For long-term based models, the inputs are the same as the LSMR.

- Long-term Memory Retrieval LSTM (LMR-LSTM): the model only has sentence encoder, query extractor, long-term memory retriever and volatility predictor four component. In the volatility predictor component, the LSTM applies to retrieved sequences.

- Long-term Memory Retrieval RIMs (LMR-RIMs): The LSTM in LMR-LSTM is replaced with RIMs. 


\begin{tabular}{|c|c|c|c|c|c|c|c|c|c|c|c|c|}
\hline \multirow{2}{*}{ Models } & \multicolumn{6}{|c|}{ SPVt1 Prediction } & \multicolumn{6}{|c|}{ SPVt22 Prediction } \\
\hline & MAPE & MAE & MSE & Precision & Recall & F1-value & MAPE & MAE & MSE & Precision & Recall & F1-value \\
\hline NSVM & 128.544 & 0.010401 & 0.000145 & 0.106 & 0.021 & 0.035 & 92.844 & 0.033257 & 0.001332 & 0.000 & 0.000 & 0.000 \\
\hline StockNet & 58.999 & 0.006608 & 0.000102 & 0.000 & 0.000 & 0.000 & 49.744 & 0.023050 & 0.000889 & 0.100 & 0.002 & 0.003 \\
\hline Mean Baseline & 131.332 & 0.010584 & 0.000148 & 0.000 & 0.000 & 0.000 & 92.817 & 0.033242 & 0.001330 & 0.000 & 0.000 & 0.000 \\
\hline NASM-LSTM & 81.081 & 0.007735 & 0.000112 & 0.079 & 0.012 & 0.020 & 79.756 & 0.030662 & 0.001433 & 0.180 & 0.170 & 0.174 \\
\hline LMR-LSTM(30) & 121.761 & 0.010733 & 0.000188 & 0.099 & 0.198 & 0.131 & 92.174 & 0.033735 & 0.001565 & 0.175 & 0.253 & 0.197 \\
\hline LSMR-LSTM(30) & 117.092 & 0.010008 & 0.000157 & 0.128 & 0.205 & 0.154 & 81.545 & 0.031132 & 0.001339 & 0.185 & 0.147 & 0.143 \\
\hline LMR-LSTM(90) & 104.591 & 0.009630 & 0.000154 & 0.113 & 0.092 & 0.094 & 79.033 & 0.030863 & 0.001343 & 0.183 & 0.138 & 0.147 \\
\hline LSMR-LSTM(90) & 100.505 & 0.009274 & 0.000147 & 0.203 & 0.162 & 0.156 & 67.839 & 0.028320 & 0.001234 & 0.165 & 0.087 & 0.089 \\
\hline NASM-RIMs & 135.390 & 0.011073 & 0.000173 & 0.139 & 0.209 & 0.166 & 90.919 & 0.034182 & 0.001683 & 0.189 & 0.283 & 0.217 \\
\hline LMR-RIMs(30) & 148.395 & 0.013331 & 0.000290 & 0.127 & 0.315 & 0.180 & 97.838 & 0.036651 & 0.001935 & 0.185 & 0.357 & 0.239 \\
\hline LSMR-RIMs(30) & 163.774 & 0.014468 & 0.000327 & 0.119 & 0.386 & 0.181 & 112.315 & 0.041028 & 0.002405 & 0.187 & 0.503 & 0.272 \\
\hline LMR-RIMs(90) & 151.072 & 0.013352 & 0.000292 & 0.120 & 0.322 & 0.174 & 98.488 & 0.037008 & 0.001952 & 0.183 & 0.388 & 0.248 \\
\hline LSMR-RIMs(90) & 169.196 & 0.014992 & 0.000358 & 0.122 & 0.393 & 0.185 & 103.940 & 0.038998 & 0.002155 & 0.175 & 0.408 & 0.245 \\
\hline
\end{tabular}

Table 1: Performance of baselines and our models to predict the SPVt1 and the SPVt22 on the testing dataset (the size of longterm memory is displayed in parentheses, 30 represents previous 30 days news, 90 represents previous 90 days news; we use boldface to label LSMR-based models).

Moreover, we compare our models with three baselines,

- NSVM [17]: a stochastic recurrent neural network framework which can formulate the temporal dynamics of volatility over time and predict the $\log$-return $x_{t}=\log \left(s_{t} / s_{t-1}\right)$ of actual prices $s_{t}$ on date $t$. Different from our model, NSVM is evaluated based on Negative Log-Likelihood (NLL). In order to compare our models with NSVM on predicting the $S P V t_{n}$, we train the model by multi-task learning. The input is the same as the short-term based models. Since $\Sigma_{t}^{x}$ represents the variance of $x_{t}, S P V t_{n}$ represents the volatility of series, it can be regarded as variance as well, so we add another linear layer on the top of $\Sigma_{t}^{x}$ and predict the $S P V t_{n}$. The joint loss is the sum of MSE and NLL. We predefine the dimensions of observable variables, and the latent variables are the same as in [17].

- StockNet [20]: a model to predict the movement of the stock based on tweets and historical prices. In order to compare the StockNet with our models, we have to modify their architecture: (1) StockNet predicts stock movements based on tweets and historical prices. In contrast, our models predict the volatility only based on news. So, we remove Market Information Encoder(MIE) component in StockNet and directly use our encoded input to replace MIE; (2) Since our models work on the regression problem, however, StockNet is applied on the classification task. We remove the softmax function on the top layer so that the model can predict volatility; (3) The objective function of StockNet contains a likelihood term and a KL term. We replace the likelihood term with Mean Square Error (MSE), so that we can train the modified StockNet successfully. The input is the same as the short-term based models.

- Mean baseline: the mean of all true values in the training and validation dataset is the prediction.

\subsection{Experimental Results}

Table 1 summarizes the results of the testing dataset in the metrics MAPE, MAE, MSE, Precision, Recall, and F1-value. Although
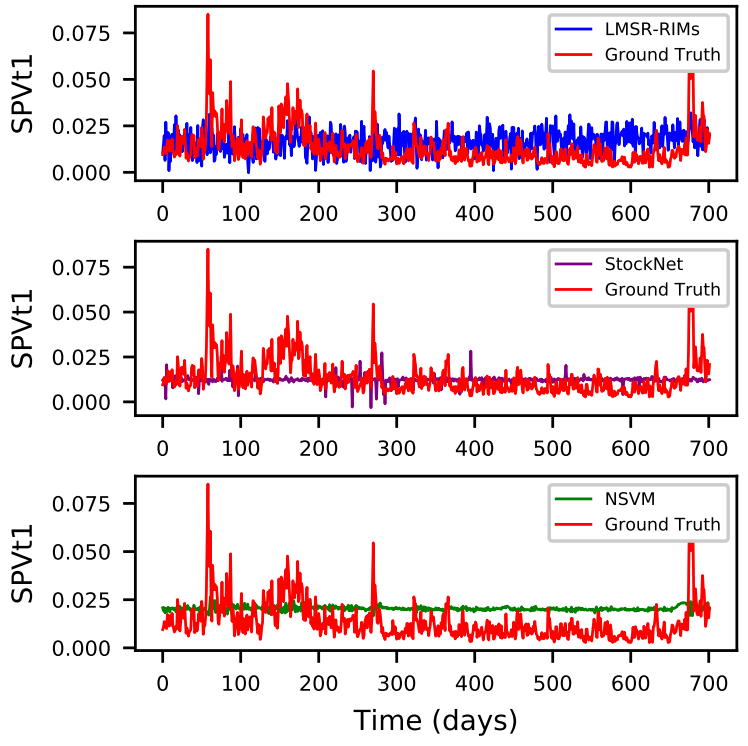

Figure 3: SPVt1 prediction results of LMSR-RIMs, StockNet, and NSVM on testing dataset visualized.

StockNet achieves the lowest MAPE, MAE, and MSE among all the models on SPVt1 and SPVt22 predictions, part of our proposed models can still beat the other two baselines. Moreover, we found that, in the testing dataset, the mean and standard deviation of SPVt1 is 0.013 and 0.009828 ; the mean and standard deviation of SPVt22 is 0.052 and 0.029 . This means that the variations of SPVt1 and SPVt22 are in a small range. If the predictions fluctuate around the mean, the models can obtain low errors, while they do not successfully fit target variables. In reality, spikes (high volatility) reflect high risks; how well the models predict the spikes are of great practical importance. Under F1-value, we can see that the models based on LSMR architecture perform better than proposed models and baselines (except LSMR-LSTM(30) and LSMR-LSTM(90) on SPVt22 prediction), and all the RIMs-based models outperform 



Figure 4: SPVt22 prediction results of LMSR-RIMs, StockNet, and NSVM on testing dataset visualized.

other models, which indeed valid the efficiency of our LSMR architecture and capability of RIMs to capture latent dynamics. In the next section, we will analyze the empirical results in detail.

For visualization, we first randomly selected one run result out of five-run for all models. Then, we plotted the predictions results of LSMR-RIMs(30), StockNet, and NSVM on the testing dataset in Figure 3 and Figure 4. Red lines in all figures are the target variables. As we can see, the predictions of StockNet and NSVM fluctuate in a small range; in contrast, LSMR-RIMs(30) demonstrates the power to predict the spikes. The plots regarding Stockett and NSVM in two figures again valid that low MAPE, MAE, and MSE can not sufficiently demonstrate the ability of models to predict volatility; therefore, it indeed convinces the necessity of levering Precision, Recall, and F1-value to evaluate model performances.

\section{ANALYSIS}

In this section, we analyze the empirical results from the following four aspects based on F1-value: (1) SPVt1 vs. SPVt22; (2) LMSR architecture and models; (3) long-term memory vs. short-term memory; (4) long-term memory size (30 vs. 90 days).

SPVt1 vs. SPVt22: SPVt1 and SPVt22 display the variation of the S\&P 500 (SP) index during 1 and 22 trading days after the anchor day separately. Since SPVt1 indicates the variation of the SP index in one day, it fluctuates more heavily than SPVt22, so that intuitively it is harder than SPVt22 task. The red lines in Figure 3 correspond true SPVt1 in the testing dataset, and the red lines in Figure 4 are true SPVt22 in the testing data. It is obvious that the plot of SPVt22 is smoother than SPVt1. In the testing dataset, for SPVt1, there are 85 out of 702 days whose true values are higher than $\mu+\sigma$; for SPVt22, 120 out of 702 days whose actual values are higher than $\mu+\sigma$. Even though SPVt22 has more spikes than SPVt1 in the testing dataset, our model still can achieve a higher F1-value on the SPVt22 task; it indeed proves SPVt1 is a tougher task.

LMSR architecture and models: In this paper, we propose a Long-term Memory Retrieval (LMR) mechanism to overcome the drawback of RNN on dealing with extremely long event sequences. As shown in Table 1, all of the LMR-based models have higher F1-value than baseline on SPVt1 prediction. For SPVt22 prediction, expect LMR-LSTM(90) is 0.027 lower than NASM-LSTM on F1value, all the other LMR-based models still outperform short-term models. The results indeed prove that the LMR mechanism can improve the efficiency of RNNs to process long-term memory by extracting event sub-sequences from long-term memory.

By incorporating short-term memory with long-term memory, we present a Long- and Short-term Memory Retrieval (LSMR) architecture, which not only utilizes the long-term memory but also takes the short-term information into account. The results of LSMRbased models are labeled in boldface in Table 1. All the LSMR-based models outperform short-term based, LMR-based models on SPVt1 prediction. For SPVt22 prediction, even though LSMR-LSTM(30)(90) can not beat NASM-LSTM, LMR-LSTM(30)(90), they still perform better than other baselines. Moreover, the LSMR-RIMs(90) and LSMR-RIMs(30) have the highest F1-value on SPVt1 prediction and SPVt22 prediction separately, and both best models are based on the LSMR architecture.

To prove the efficiency of RIMs, we compared LMR-LSTM with LMR-RIMs, LSMR-LSTM with LSMR-RIMs separately. It can be seen from the data in Table 1 that all RIMs-based models perform better than LSTM-based models. It indeed proves that RIMs act independently, and communicate only sparingly through the bottleneck of attention, have the potential to detect and capture more underlying dynamics from data.

Long-term memory vs. short-term memory: In Table 1, all the models utilizing long-term memory achieve higher F1-value than short-term based models (except NASM-RIMs). In order to remove the influence of RIMs, we also compare NASM-RIMs with LMR-RIMs and LSMR-RIMs. The results show that LMR-RIMs and LSMR-RIMs outperform NASM-RIMs, confirming that long-term memory is useful to predict volatility in our tasks.

Long-term memory size (30 vs. 90 days): We conducted experiments about different long-term memory size. Although LSMRRIMs(90) has the highest F1-value on the SPVt1 prediction task, there is no sufficient evidence to illustrate that 90 days' memory is better than 30 days. However, since 90 days' memory is only the medium-term memory, we will systematically study the exact long-term memory in the future.

\section{CONCLUSION AND FUTURE WORK}

In this paper, we first build a novel news dataset from the Wall Street Journal (WSJ) for predicting the short-term and mid-term volatility. 
Next, we propose a long- and short-term memory retrieval (LSMR) architecture, which efficiently captures long-term information by a long-term memory retrieval mechanism, then applies recurrent neural networks (LSTM or RIMs) to make predictions. Extensive experiments are conducted on the WSJ dataset that exhibit the efficiency of the long-term memory retrieval mechanism to handle extremely long memory, the capability of RIMs to capture dynamics, and the superior performance of our models in predicting highly volatile scenarios.

Our future works include improving the LSMR architecture by introducing reasoning mechanisms, attempting other embedding techniques, systematic study of long-term memory size, and conducting experiments on predicting long-term volatility and comparing the results with short- and mid-term volatility.

\section{REFERENCES}

[1] John Black, Nigar Hashimzade, and Gareth Myles. 2012. A dictionary of economics Oxford university press.

[2] Tim Bollerslev. 1986. Generalized Autoregressive Conditional Heteroskedasticity. JOURNAL OF ECONOMETRICS 31 (1986), 307-327.

[3] Junyoung Chung, Caglar Gulcehre, Kyunghyun Cho, and Yoshua Bengio. 2014 Empirical evaluation of gated recurrent neural networks on sequence modeling. In NIPS 2014 Workshop on Deep Learning, December 2014.

[4] Jacob Devlin, Ming-Wei Chang, Kenton Lee, and Kristina Toutanova. 2019. BERT: Pre-training of Deep Bidirectional Transformers for Language Understanding. In Proceedings of the 2019 Conference of the North American Chapter of the Association for Computational Linguistics: Human Language Technologies, Volume 1 (Long and Short Papers). Association for Computational Linguistics, Minneapolis, Minnesota, 4171-4186. https://doi.org/10.18653/v1/N19-1423

[5] Xiao Ding, Yue Zhang, Ting Liu, and Junwen Duan. 2014. Using Structured Events to Predict Stock Price Movement: An Empirical Investigation. In Proceedings of the 2014 Conference on Empirical Methods in Natural Language Processing (EMNLP). Association for Computational Linguistics, Doha, Qatar, 1415-1425. https://doi.org/10.3115/v1/D14-1148

[6] Xiao Ding, Yue Zhang, Ting Liu, and Junwen Duan. 2015. Deep Learning for Event-Driven Stock Prediction. In Proceedings of the 24th International Conference on Artificial Intelligence (Buenos Aires, Argentina) (IfCAI'15). AAAI Press, 2327-2333.

[7] Robert F Engle. 1982. Autoregressive Conditional Heteroscedasticity with Estimates of the Variance of United Kingdom Inflation. Econometrica 50, 4 (July 1982), 987-1007. https://ideas.repec.org/a/ecm/emetrp/v50y1982i4p987-1007.html

[8] Robert F. Engle. 1982. Autoregressive Conditional Heteroscedasticity with Estimates of the Variance of United Kingdom Inflation. Econometrica 50, 4 (1982) 987-1007. http://www.jstor.org/stable/1912773

[9] Xavier Glorot and Yoshua Bengio. 2010. Understanding the difficulty of training deep feedforward neural networks. In Proceedings of the Thirteenth International Conference on Artificial Intelligence and Statistics (Proceedings of Machine Learning Research), Yee Whye Teh and Mike Titterington (Eds.), Vol. 9. PMLR, Chia Laguna Resort, Sardinia, Italy, 249-256. http://proceedings.mlr.press/v9/glorot10a.html

[10] Anirudh Goyal, Alex Lamb, Jordan Hoffmann, Shagun Sodhani, Sergey Levine, Yoshua Bengio, and Bernhard Schölkopf. 2019. Recurrent Independent Mechanisms. arXiv:cs.LG/1909.10893

[11] Steven L. Heston. 1993. A closed-form solution for options with stochastic volatility with applications to bond and currency options. Review of Financial Studies 6 (1993), 327-343.

[12] Sepp Hochreiter and Jürgen Schmidhuber. 1997. Long Short-Term Memory. Neural Computation 9, 8 (1997), 1735-1780.

[13] Ziniu Hu, Weiqing Liu, Jiang Bian, Xuanzhe Liu, and Tie-Yan Liu. 2017. Listening to Chaotic Whispers: A Deep Learning Framework for News-oriented Stock Trend Prediction. https://doi.org/10.1145/3159652.3159690

[14] Ziniu Hu, Weiqing Liu, Jiang Bian, Xuanzhe Liu, and Tie-Yan Liu. 2018. Listening to chaotic whispers: A deep learning framework for news-oriented stock trend prediction. In Proceedings of the eleventh ACM international conference on web search and data mining. 261-269.

[15] John C Hull and Alan White. 1987. The Pricing of Options on Assets with Stochastic Volatilities. Journal of Finance 42, 2 (1987), 281-300. https://EconPapers. repec.org/RePEc:bla:jfinan:v:42:y:1987:i:2:p:281-300

[16] Diederik P. Kingma and Jimmy Ba. 2014. Adam: A Method for Stochastic Optimization. http://arxiv.org/abs/1412.6980 cite arxiv:1412.6980Comment: Published as a conference paper at the 3rd International Conference for Learning Representations, San Diego, 2015.
[17] Rui Luo, Weinan Zhang, Xiaojun Xu, and Jun Wang. 2018. A Neural Stochastic Volatility Model. AAAI Conference on Artificial Intelligence (2018). https://aaai. org/ocs/index.php/AAAI/AAAI18/paper/view/17331

[18] Navid Rekabsaz, Mihai Lupu, Artem Baklanov, Alexander Dür, Linda Andersson, and Allan Hanbury. 2017. Volatility Prediction using Financial Disclosures Sentiments with Word Embedding-based IR Models. In Proceedings of the 55th Annual Meeting of the Association for Computational Linguistics (Volume 1: Long Papers). Association for Computational Linguistics, Vancouver, Canada, 1712-1721. https://doi.org/10.18653/v1/P17-1157

[19] Thomas Wolf, Lysandre Debut, Victor Sanh, Julien Chaumond, Clement Delangue, Anthony Moi, Pierric Cistac, Tim Rault, R'emi Louf, Morgan Funtowicz, and Jamie Brew. 2019. HuggingFace's Transformers: State-of-the-art Natural Language Processing. ArXiv abs/1910.03771 (2019).

[20] Yumo Xu and Shay B. Cohen. 2018. Stock Movement Prediction from Tweets and Historical Prices. In Proceedings of the 56th Annual Meeting of the Association for Computational Linguistics (Volume 1: Long Papers). Association for Computational Linguistics, Melbourne, Australia, 1970-1979. https://doi.org/10.18653/v1/P181183

[21] Linyi Yang, Tin Lok James Ng, Barry Smyth, and Riuhai Dong. 2020. HTML: Hierarchical Transformer-Based Multi-Task Learning for Volatility Prediction. In Proceedings of The Web Conference 2020 (Taipei, Taiwan) (WWW'20). Association for Computing Machinery, New York, NY, USA, 441-451. https://doi.org/10. 1145/3366423.3380128

[22] Linyi Yang, Zheng Zhang, Su Xiong, Lirui Wei, James Ng, Lina Xu, and Ruihai Dong. 2018. Explainable Text-Driven Neural Network for Stock Prediction. In 5th IEEE International Conference on Cloud Computing and Intelligence Systems, CCIS 2018, Nanjing, China, November 23-25, 2018. IEEE, 441-445. https://doi.org/ 10.1109/CCIS.2018.8691233 